https://doi.org/10.1590/198053146162

\title{
RESPETO E IGUALDAD DE GÉNERO EN LA FORMACIÓN INICIAL DOCENTE
}

\author{
Rocío Serrano Rodríguez' \\ Elisa Pérez Gracia" \\ Monserrat Bustamante Chan'"I \\ Antonio Carpio Camargo Iv
}

\section{Resumen}

Se presentan los resultados del análisis de las opiniones de 353 estudiantes del Máster de Formación del Profesorado de Educación Secundaria de la Universidad de Córdoba (España) sobre la competencia transversal centrada en planificar espacios de aprendizaje que favorezcan el respeto y la igualdad de género. Mediante un cuestionario escala Likert se han recogido las aportaciones con relación al nivel de desarrollo y la importancia atribuida a tal competencia. Los resultados muestran la existencia de diferencias entre géneros y macro-áreas de conocimiento en el caso del nivel de desarrollo adquirido. Consideramos que es necesario profundizar en los factores que propician estas diferencias y en la evolución de los distintos estilos de aprendizaje por titulación universitaria y género. Esto permitiría una mayor comprensión de los modos de aprendizaje del alumnado y como consecuencia, una mejora de su proceso formativo.

\section{RELACIONES DE GÉNERO • FORMACIÓN DEL PROFESORADO • COMPETENCIA PROFESIONAL}

\section{GENDER RESPECT AND EQUALITY IN THE INITIAL TEACHER TRAINING}

\section{Abstract}

This paper presents the results of the analysis of the opinions of 353 students enrolled in the Master's degree in Secondary Education Teacher Training at the University of Córdoba (Spain) regarding cross-curricular competences focused on organizing learning spaces that promote gender respect and equality. Through a Likert scale questionnaire, data have been collected in relation to the level of development and the importance conferred to each competence. The results show differences in terms of gender and macro-areas of knowledge in the case of the level of development. We believe that it is necessary to study in depth the factors that favor these differences as well as the evolution of the diverse learning styles by university degree and gender. This would allow a greater understanding of the learning methods of the students and therefore, an improvement of their training process.

\section{GENDER RELATIONS • TEACHER TRAINING - PROFESSIONAL COMPETENCE}

I Universidad de Córdoba, Córdoba, España; https://orcid.org/0000-0002-9447-9336; rocio.serrano@uco.es

II Universidad Loyola, Sevilla, España; https://orcid.org/0000-0002-6219-7203; eperez@uloyola.es

III Universidad Tecnológica ECOTEC, Guayaquil, Ecuador; https://orcid.org/0000-0002-7953-6021; mbustamante@ecotec.edu.ec

IV Instituto de Investigación en Recursos Cinegéticos (IREC), Ciudad Real, España; https://orcid.org/0000-0002-6989-970X; antonio.carpio@unesum.edu.ec 


\section{RESPECT ET ÉGALITÉ DES SEXES DANS LA FORMATION INITIALE DE L'ENSEIGNEMENT}

\section{Résumé}

Les résultats de l'analyse des opinions de 353 étudiants du Master en formation a l'enseignement secondaire de l'Universidad de Córdoba (Espagne) sur les compétences transversales axées sur la planification d'espaces d'apprentissage favorisant le respect et l'égalité des sexes sont présentés. Un questionnaire à l'échelle de Likert a permis de collecter des contributions relatives au niveau de développement et à l'importance attribuée à cette competence. Les résultats montrent l'existence de différences entre les genres et les macrodomaines de connaissance dans le cas du niveau de développement acquis. Nous pensons qu'il est nécessaire d'étudier en profondeur les facteurs qui favorisent ces différences et l'évolution des différents styles d'apprentissage en fonction du diplôme universitaire et du sexe. Cela permettrait une meilleure compréhension des méthodes d'apprentissage des étudiants et, par conséquent, une amélioration de leur processus de formation.

RELATIONS DE GENRE • FORMATION DES ENSEIGNANTS • COMPÉTENCE PROFESSIONNELLE

\section{RESPEITO E IGUALDADE DE GÊNERO NO ENSINO DA FORMAÇÃO INICIAL}

\section{Resumo}

São apresentados os resultados da análise das opiniões de 353 alunos do Mestrado de Formação dos Professores de Ensino Médio da Universidade de Córdoba (Espanha) sobre competência transversal focada no planejamento de espaços de aprendizagem que promovam o respeito e a igualdade de gênero. Através de um questionário na escala Likert, foram coletadas contribuições em relação ao nível de desenvolvimento e à importância atribuída a tal competência. Os resultados mostram a existência de diferenças entre gêneros e macroáreas de conhecimento no caso do nível de desenvolvimento adquirido. Acreditamos que é preciso aprofundar a investigação sobre os fatores que favorecem essas diferenças e a evolução dos diferentes estilos de aprendizagem por nível universitário e gênero. Isso permitiria uma maior compreensão dos métodos de aprendizagem dos alunos e, assim, uma melhoria de seu processo formativo.

RELAÇÕES DE GÊNERO • FORMAÇÃO DE PROFESSORES • COMPETÊNCIA PROFISSIONAL 
- L BANCO MUNDIAL, EN SU INFORME ANUAL SOBRE EL DESARROLLO DE LA EDUCACIÓN,

destaca entre sus prioridades actuales garantizar y promover oportunidades de aprendizaje que favorezcan la igualdad de género, además de la mejora de la Educación Superior y la formación del profesorado (WORLD BANK, 2018).

Según Severiens y Ten Dam (1994) los trabajos sobre educación y género se vienen planteando desde la década de los setenta. Sobre todo, aportes centrados en cómo influye el género en el fracaso escolar, la elección y éxitos de los estudios, los estilos y los enfoques de aprendizaje (MILLER; FINLEY; MCKINLEY, 1990; WILSON; SMART; WATSON, 1996; LEDER; FORGASZ, 2018). Sin embargo, el tema de la influencia del género en el diseño de prácticas de aprendizaje que favorezcan el respeto y la igualdad de género (GARCÍA et al., 2011) ha sido un tema poco estudiado en la literatura.

Desde este contexto, nos surgen dos inquietudes que abordamos en este estudio; por un lado, la preocupación por la formación inicial del profesorado en género; de otro lado, las creencias y actitudes que manifiesta el alumnado sobre la competencia transversal centrada en diseñar y desarrollar espacios de aprendizaje que favorezcan el respeto y la igualdad de género. Distinguiendo entre el nivel de desarrollo y la importancia que se les concede a tales competencias para el desarrollo de la profesionalidad docente. 
Es importante recordar que en los planes de estudios universitarios se recogen indicaciones muy específicas sobre la inclusión de la perspectiva de género en la formación básica del alumnado. Por tanto, desarrollar este tipo de investigaciones permitirá analizar las fortalezas y debilidades del proceso actual de formación inicial docente del profesorado de secundaria, y con ello, la posibilidad de elaborar propuestas curriculares bien fundamentadas, que ayuden a mejorar la FIPS en el futuro.

\section{EL ESPACIO EUROPEO DE EDUCACIÓN SUPERIOR Y LA FORMACIÓN INICIAL DEL PROFESORADO DE SECUNDARIA}

A nivel internacional y nacional se evidencia un profundo interés en la formación inicial del profesorado y el desarrollo de la Identidad Profesional Docente (IZADINIA, 2016; LORENZO VICENTE; MUÑOZ GALIANO; BEAS MIRANDA, 2015; LE HUU NGHIA; NGOC TAI, 2017; FERRADA; VILLENA; DEL PINO, 2018). Aunque como indican Day y Gu (2012), la formación del profesorado de secundaria basada en el desarrollo de competencias docentes es un reto complicado para los formadores del profesorado.

En elestudio de Sánchez, LópezyFernández(2010)mostraron la importancia de las competencias transversales en todas las titulaciones universitarias. Según Zabalza (2011), la inclusión de competencias transversales en todos los itinerarios educativos universitarios ha demostrado ser tremendamente valiosa para la educación integral de los y las estudiantes e implica una gran relevancia para los y las profesionales, ya que facilita su integración y participación en el mercado laboral. Es deber de los y las docentes integrar las competencias transversales correspondientes a cada materia en sus actividades educativas para facilitar el desarrollo de su alumnado (VILLA; POBLETE, 2011).

De acuerdo con Arribas, Manrique y Tabernero (2016) es vital llevar a cabo un análisis en profundidad de las competencias adquiridas para poder evaluar y mejorar la empleabilidad de los graduados y las graduadas. Como tal, en el documento sobre las políticas docentes de la UNESCO (2017), se presta especial atención a la formación docente de calidad y en particular, a la preparación inicial de maestros y maestras y docentes de secundaria. Concretamente, se definen políticas encaminadas a la evaluación del profesorado y como tal, a la regulación de programas formativos que incluyan el conjunto de competencias transversales definidas (GONZÁLEZ; WAGENAAR, 2006).

\section{DISEÑAR Y DESARROLLAR ESPACIOS DE APRENDIZAJE QUE FAVOREZCAN EL RESPETO Y LA IGUALDAD DE GÉNERO}

Partiendo de la idea generalizada, como indica Forbes y Davis (2010), de que es imprescindible conocer las actitudes de los y las docentes en formación y en ejercicio, ya que la introducción de innovaciones educativas se encuentra condicionada por las actitudes de este colectivo hacia el conocimiento de qué enseñar y cómo. Podemos encontrarnos que actitudes negativas del profesorado hacia el desarrollo de cualquier competencia en su alumnado conlleva una 
disminución en el tiempo que se dedica a esta frente a otras de un mayor nivel de seguridad.

En relación con lo anterior, Goldman y Warren (1973), Watkins y Hattie (1985) y Delgado y Prieto (1993), entre otros, señalaron que las diferentes carreras universitarias plantean contextos de aprendizaje heterogéneos y como consecuencia, distintos tipos de conductas y enfoques de aprendizaje diferentes. Sin embargo, en ninguno de estos estudios se incluyen diferencias de género. Por el contrario, en la investigación de Cano (2000) se concluye la existencia de una relación significativa entre el género del alumnado y la interpretación que se realiza de las estrategias y estilos de aprendizaje, así como la influencia ejercida por el tipo de carrera estudiada. En esta misma línea, encontramos el estudio desarrollado por Harlen (2015), donde se muestran que esas actitudes están en parte condicionadas por las experiencias previas durante su formación anterior, influyendo en su concepción y en cómo abordarla en un futuro próximo en el aula.

De igual forma, González (2016) afirma que la resistencia del profesorado a los temas de género es consecuencia de su ausencia en la preparación inicial recibida. El profesorado no es consciente y no valora la importancia de su influencia y de la proyección de sus creencias con respecto al género en su práctica docente. Esen (2013) concluye su investigación haciendo hincapié en que esta resistencia a la inclusión de nuevas estrategias que alberguen la igualdad de género en clase desaparecería si las políticas de formación docente incluyeran la sensibilidad de género en el marco de un docente de calidad, asegurándose que las instituciones educativas cumplan con estos criterios.

De manera más concreta, en el estudio desarrollado por Walton y sus colaboradores (2018) sobre los enfoques pedagógicos y las estrategias utilizadas por los y las futuras docentes en formación sobre justicia social y relacionadas con el género, la raza, la sexualidad, las necesidades educativas especiales, el estatus socioeconómico y la religión, se concluye que todavía hay una escasa formación en estos contenidos y se destaca la necesidad de seguir reforzando la formación con programas que aborden la enseñanza para la justicia social con prácticas pedagógicas relacionadas con la igualdad de género (UKPOKODU, 2016). Resultados muy en consonancia con los aportes de García, Sala, Rodríguez y Sabuco (2013), donde destacan la necesidad de una capacitación profesional para abordar, desde la educación, una cultura de género inmersa desde la práctica y los contextos escolares.

A pesar de todo, algunos investigadores han sugerido que las diferencias de género podrían ser el resultado de diferentes actitudes hacia la situación de la prueba (por ejemplo, ansiedad ante la prueba o evitación del rendimiento) en lugar de diferencias reales en el conocimiento o la competencia (LINDBERG et al., 2010; HANNON, 2012). En esa misma línea, Leder y Forgasz (2018) discuten cómo el formato y el propósito de la evaluación influye en los resultados finales del alumnado de acuerdo con el género. 
Finalmente, como demuestran Díaz de Grenu y Anguita (2017) en el ámbito de la formación del profesorado existe un sesgo de género en los discursos y prácticas del profesorado, que, como consecuencia, refuerzan la desigualdad. La práctica docente en torno al género responde en su mayoría a inquietudes que ponen de manifiesto las mujeres en el diseño de sus programaciones. Por ello, todo el profesorado debería pasar por un proceso de autorreflexión y toma de conciencia de sus prácticas como prevención contra esa ceguera de género (REBOLLO CATALÁN; VEGA CARO; GARCÍA-PEREZ, 2011).

\section{DISEÑO METODOLÓGICO}

\section{OBJETIVOS}

De forma global, la finalidad de la presente investigación ha sido conocer cómo valora el futuro profesorado la formación adquirida y la importancia atribuida a diseñar y desarrollar espacios de aprendizaje que favorezcan el respeto y la igualdad de género.

De manera más concreta, se establecieron los siguientes objetivos específicos:

1. Conocer las ideas previas del alumnado sobre el nivel de desarrollo y la importancia atribuida a tal competencia en relación con el conjunto global de competencias transversales.

2. Analizar la relación entre la importancia atribuida y el nivel de desarrollo adquirido en dicha competencia.

3. Determinar la influencia de factores como el género, la edad y el área de conocimiento.

\section{Participantes}

En este estudio participaron una muestra total de 353 estudiantes procedentes de los cursos académicos 2011-2013, lo que supuso el 92\% de la población que accedió al Máster de Formación del Profesorado de Secundaria en la Universidad de Córdoba (España). El rango principal de edad oscila entre los $20-25$ años (43,9\%), seguido del rango de edad $26-30(36,5 \%)$ y el resto $(19,6 \%)$ se corresponde con alumnado de edad superior a 30 años. En relación al género, el $53,8 \%$ de las aspirantes a futuras docentes son mujeres.

En la Tabla 1 podemos ver la distribución de los y las participantes según el área de conocimiento de acceso al MAES: Ciencias Experimentales (Física y Química, Biología y Geología, Ciencias de la Salud y el Deporte), Ciencias Instrumentales (Tecnología-Dibujo, Informática y Matemáticas), Ciencias Sociales (Historia-Geografía, Economía-Empresa-Comercio, Formación y Orientación Laboral, Orientación Educativa) y Humanidades (Idioma Extranjero, Lengua y Literatura Española y Música) 
TABLA 1

DISTRIBUCIÓN DE LA MUESTRA SEGÚN ÁREAS DE CONOCIMIENTO

\begin{tabular}{l|c|c}
\hline \multicolumn{1}{c|}{ ÁREA DE CONOCIMIENTO } & FRECUENCIA & PORCENTAJE \\
\hline Ciencias Experimentales(CE) & 135 & 38,2 \\
\hline Ciencias Instrumentales (Cl) & 85 & 24,1 \\
\hline Ciencias Sociales (CS) & 30 & 8,5 \\
\hline Humanidades (HUM) & 103 & 29,2 \\
\hline Total & 353 & 100,0 \\
\hline
\end{tabular}

Fuente: Elaboración propia.

\section{Instrumento}

Para el desarrollo de la investigación se diseñó un cuestionario escala Likert con una escala de valoración que incluye cinco categorías posibles que oscilan desde 1 (mínimo) a 5 (máximo). El cuestionario se organiza en una sección inicial de datos generales (edad, género, año de finalización de estudios y especialidad del máster) y el resto de las preguntas siguientes aparecen distribuidas en tres secciones centradas en valorar: las expectativas de los objetivos, las competencias generales y las competencias establecidas para el prácticum en el MAES. El cuestionario pasó por un proceso de validación conceptual, de compresión y de constructo, alcanzando un grado muy elevado de fiabilidad interna (Alfa Cronbach 0,956) (SERRANO; PONTES, 2015).

Centrándonos en el objetivo de esta investigación, en la Cuadro 1 se recoge el conjunto de competencias transversales previstas para el MAES (ESPAÑA, 2007) donde se incluye la competencia: Diseñar y desarrollar espacios de aprendizaje que favorezcan el respeto y la igualdad de género.

\section{CUADRO 1}

\section{COMPETENCIAS TRANSVERSALES MAES}

\begin{tabular}{|c|c|}
\hline ÍTEMS & ENUNCIADO \\
\hline 1 & $\begin{array}{l}\text { Conocer los contenidos curriculares de las materias relativas a la especialización docente } \\
\text { correspondiente. }\end{array}$ \\
\hline 2 & Planificar, desarrollar y evaluar el proceso de enseñanza y aprendizaje. \\
\hline 3 & $\begin{array}{l}\text { Buscar, obtener, procesar y comunicar información (oral, impresa, audiovisual, digital o } \\
\text { multimedia), acreditando un manejo adecuado de las Tics y el dominio de una segunda } \\
\text { lengua en los procesos de comunicación. }\end{array}$ \\
\hline 4 & $\begin{array}{l}\text { Concretar el currículo que se vaya a implantar en un centro docente, desarrollando y } \\
\text { aplicando metodologías didácticas tanto grupales como personalizadas, adaptadas a la } \\
\text { diversidad de los estudiantes. }\end{array}$ \\
\hline 5 & $\begin{array}{l}\text { Diseñar y desarrollar espacios de aprendizaje que favorezcan el respeto y la igualdad de } \\
\text { género. }\end{array}$ \\
\hline 6 & $\begin{array}{l}\text { Adquirir estrategias para estimular el esfuerzo del estudiante y promover su capacidad } \\
\text { para aprender por sí mismo y con otros. }\end{array}$ \\
\hline 7 & $\begin{array}{l}\text { Conocer los procesos de interacción y comunicación en el aula, para fomentar el } \\
\text { aprendizaje y la convivencia en el aula, y abordar problemas de disciplina y resolución de } \\
\text { conflictos. }\end{array}$ \\
\hline 8 & Diseñar y realizar actividades formales y no formales. \\
\hline 9 & Conocer la normativa y la organización institucional del sistema educativo. \\
\hline
\end{tabular}




(Continuação)
\begin{tabular}{|c|l|}
\hline ÍTEMS & \multicolumn{1}{c|}{ ENUNCIADO } \\
\hline 10 & Conocer y analizar las características históricas de la profesión docente. \\
\hline 11 & $\begin{array}{l}\text { Informar y asesorar a las familias acerca del proceso de enseñanza y aprendizaje y sobre la } \\
\text { orientación personal, académica y profesional de sus hijos. }\end{array}$ \\
\hline 12 & $\begin{array}{l}\text { Fomentar el espíritu crítico, reflexivo, emprendedor y los hábitos de búsqueda activa de } \\
\text { empleo }\end{array}$ \\
\hline 13 & $\begin{array}{l}\text { Favorecer y garantizar el respeto a los Derechos Humanos y a los principios de } \\
\text { accesibilidad universal, igualdad, no discriminación y fomento de los valores democráticos } \\
\text { y de la cultura de paz. }\end{array}$ \\
\hline
\end{tabular}

Fuente: España (2007).

\section{Procedimiento y análisis de datos}

La recogida de datos se realizó mediante la plataforma Moodle en un seminario virtual incluido en el módulo de "Practicum". Estos datos se han codificado y se han aplicado varios tratamientos estadísticos con ayuda del paquete informático SPSS V.22.

Como se ha anticipado, en este estudio solo se procedió a evaluar la competencia sobre el diseño y desarrollo de espacios de aprendizaje que favorezcan el respeto y la igualdad de género, a través de sus dos subcategorías: nivel de desarrollo alcanzado durante la formación recibida y la importancia que los futuros docentes atribuyen a tal competencia. Para conocer en qué nivel valora el alumnado el desarrollo adquirido y la importancia atribuida a esta competencia, se aplicaron pruebas descriptivas.

En segundo lugar, se realizó un análisis de correlación (Pearson) para calcular si existe una relación entre los valores que los alumnos asignan a la importancia y el nivel de desarrollo atribuido al diseño y desarrollo de espacios de aprendizaje que favorezcan el respeto y la igualdad de género.

Finalmente, con el objetivo de evaluar si existen diferencias en la percepción que el alumnado tiene sobre la importancia y el nivel de desarrollo adquirido en esta competencia entre género, edad y área de conocimiento, se utilizaron dos análisis de la varianza (ANOVA). Este análisis permite evaluar las diferencias en el valor de las variables respuestas: importancia y nivel de desarrollo (5 niveles) en función de los factores a evaluar: género (2 niveles), edad ( 3 niveles) y macroárea del conocimiento ( 2 niveles). En este análisis solo se incluyeron los factores fijos, ya que es confuso comparar la importancia relativa de las interacciones en una ANOVA multifactorial cuando hay más de un factor significativo encontrado (GRAHAM; EDWARDS, 2001). En ambos, se utilizó la prueba de diferencia mínima significativa de Fisher (prueba de LSD) para verificar las diferencias entre el nivel de las variables categóricas y para ilustrarlas. Los análisis estadísticos se realizaron empleando el software InfoStats (BALZARINI et al., 2002).

\section{RESULTADOS}

A continuación, presentamos los resultados de acuerdo a los objetivos específicos planteados en el estudio. 


\section{IDEAS PREVIAS SOBRE EL NIVEL DE DESARROLLO Y LA IMPORTANCIA ATRIBUIDA}

Con objeto de simplificar el análisis descriptivo se ha realizado una agrupación por los extremos de las cinco categorías de respuesta de los diferentes ítems, definiendo los siguientes niveles: I (nada o poco de acuerdo), II (acuerdo intermedio) y III (bastante o muy de acuerdo).

Como podemos observar en la Tabla 2, existe un acuerdo bastante elevado entre el alumnado del Máster MAES en cuanto a valorar como importantes todas las competencias transversales de su formación. Son valores medios que oscilan entre 3,75 (ítem 10) y 4,48 (ítems 2 y 6). Sin embargo, en relación al nivel de desarrollo que el alumnado percibe haber adquirido durante su formación, existe una mayor heterogeneidad de opiniones. Los niveles medios son más bajos que en la importancia atribuida. Unos valores medios que oscilan entre 2,92 (ítem 12) y 3,44 (ítem 9).

\section{TABLA 2}

ANÁLISIS DE FRECUENCIAS SOBRE EL NIVEL DE DESARROLLO Y LA IMPORTANCIA ATRIBUIDA

\begin{tabular}{l|c|c|c|c|c|c|c|c|c|c|c|c|c}
\hline & \multicolumn{10}{c}{ Competencias transversales } \\
\hline Nive/ de desarrol/o & $\mathbf{1}$ & $\mathbf{2}$ & $\mathbf{3}$ & $\mathbf{4}$ & $\mathbf{5}$ & $\mathbf{6}$ & $\mathbf{7}$ & $\mathbf{8}$ & $\mathbf{9}$ & $\mathbf{1 0}$ & $\mathbf{1 1}$ & $\mathbf{1 2}$ & $\mathbf{1 3}$ \\
\hline I (Nada) & 17,8 & 17,3 & 18,7 & 22,9 & 26,1 & 24,6 & 26,3 & 28,3 & 16,4 & 21,0 & 28,7 & 36,8 & 21,8 \\
\hline II (intermedio) & 35,4 & 37,4 & 32,3 & 42,2 & 33,7 & 38,8 & 34,3 & 34,0 & 37,9 & 35,7 & 33,1 & 31,2 & 33,4 \\
\hline III (bastante) & 46,7 & 45,3 & 49,0 & 34,8 & 40,2 & 36,5 & 39,4 & 37,7 & 45,6 & 43,3 & 34,2 & 32,0 & 44,8 \\
\hline Media & 3,4 & 3,4 & 3,4 & 3,2 & 3,2 & 3,2 & 3,2 & 3,1 & 3,4 & 3,3 & 3,0 & 2,9 & 3,4 \\
\hline Desviación & 1,04 & 1,00 & 1,01 & 1,03 & 1,09 & 1,08 & 1,07 & 1,06 & 1,00 & 1,02 & 1,14 & 1,20 & 1,14 \\
\hline
\end{tabular}

\begin{tabular}{l|c|c|c|c|c|c|c|c|c|c|c|c|c|c|c}
\hline \multicolumn{2}{l|}{ Importancia atribuida } & $\mathbf{1}$ & $\mathbf{2}$ & $\mathbf{3}$ & $\mathbf{4}$ & $\mathbf{5}$ & $\mathbf{6}$ & $\mathbf{7}$ & $\mathbf{8}$ & $\mathbf{9}$ & $\mathbf{1 0}$ & $\mathbf{1 1}$ & $\mathbf{1 2}$ & \multicolumn{2}{|c}{13} \\
\hline I (Nada) & 3,1 & 3,0 & 2,8 & 2,5 & 3,7 & 3,7 & 4,8 & 4,8 & 4,8 & 9,1 & 3,4 & 5,4 & 2,8 \\
\hline II (intermedio) & 9,6 & 7,1 & 11,3 & 10,5 & 13,3 & 6,2 & 6,2 & 11,0 & 15,9 & 30,3 & 12,2 & 13,9 & 10,2 \\
\hline III (bastante) & 87,3 & 89,9 & 85,8 & 87,0 & 83,0 & 90,1 & 89,0 & 84,1 & 79,3 & 60,6 & 84,4 & 80,7 & 87,0 \\
\hline Media & 4,5 & 4,5 & 4,3 & 4,4 & 4,3 & 4,5 & 4,4 & 4,2 & 4,2 & 3,8 & 4,3 & 4,3 & 4,4 \\
\hline Desviación & 0,85 & 0,79 & 0,81 & 0,81 & 0,89 & 0,81 & 0,85 & 0,87 & 0,89 & 0,96 & 0,83 & 0,97 & 0,83 \\
\hline
\end{tabular}

Fuente: Elaboración propia.

De acuerdo al primero de los objetivos específicos planteados (conocer las ideas previas del alumnado sobre el nivel de desarrollo y la importancia atribuida a tal competencia en relación con el conjunto global de competencias transversales), en la Figura 1 podemos observar cómo la competencia cinco ocupa el octavo lugar en relación al resto. Sin embargo, cuando se trata de valorar el nivel de desarrollo que alumnado considera haber adquirido, está competencia gana una posición (séptimo lugar).

De manera general, podemos indicar que, tanto en la importancia como en el nivel, desarrollar espacios de aprendizaje que tengan en cuenta la igualdad 
de género se encuentra en una posición intermedia con relación al resto de competencias transversales.

\section{FIGURA 1}

COMPETENCIAS ORDENADAS DE MAYOR A MENOR MEDIA

nédia Nivel de desarrollo

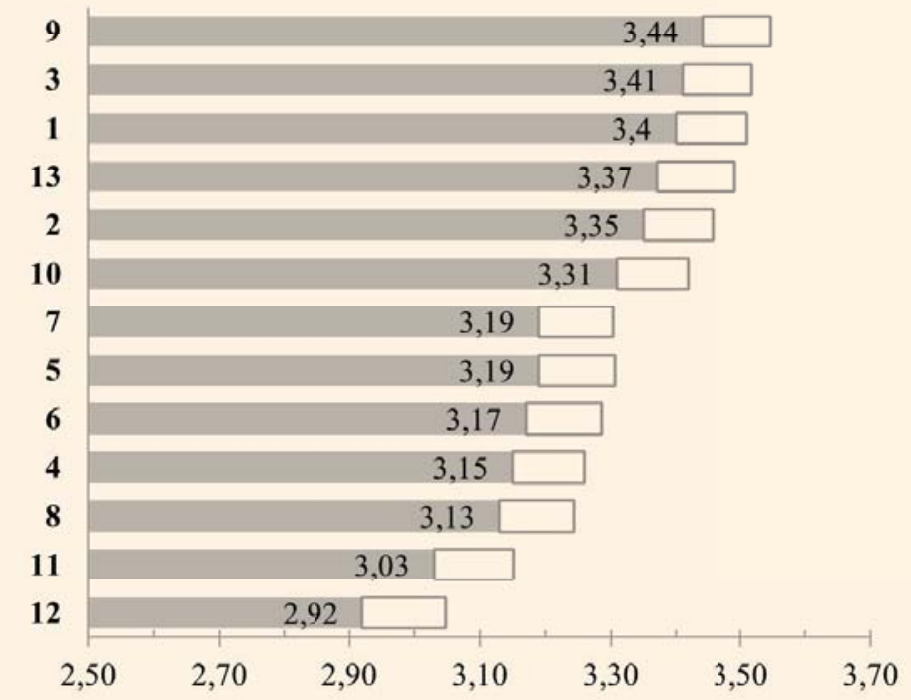

Puntuciones obtenidas en la escala Likert

" Média Importancia atribuida

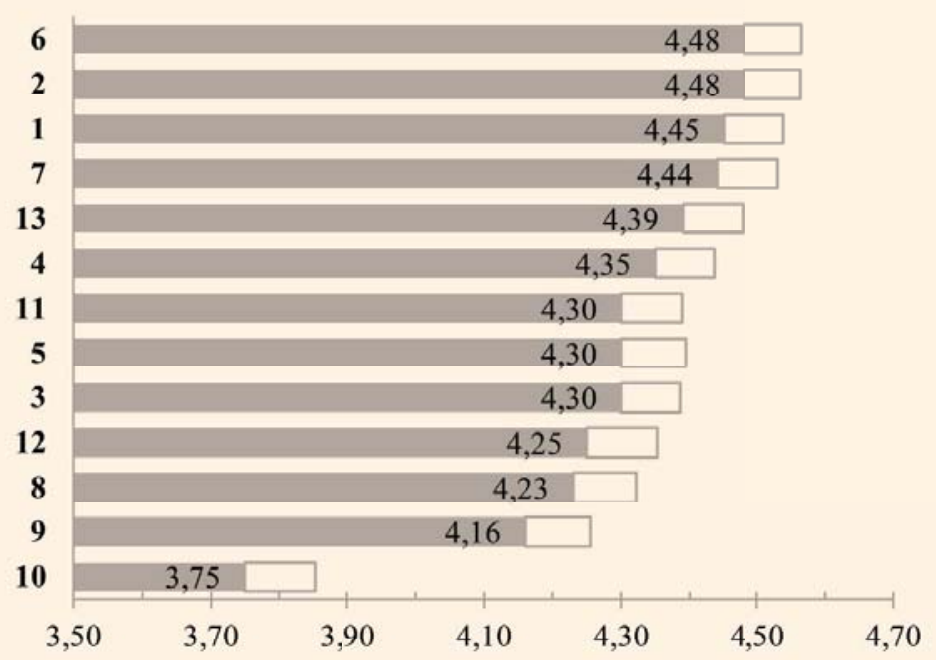

Puntuaciones obtenidas en la escala Likert

Fuente: Elaboración propia.

\section{RELACIÓN ENTRE LA IMPORTANCIA ATRIBUIDA Y EL NIVEL DE DESARROLLO ADQUIRIDO}

La correlación de Pearson muestra una relación significativa y positiva entre ambas variables (nivel e importancia) (Pearson $=0,21 ; \mathrm{p}<0,0001$ ) . 


\section{INFLUENCIA DE FACTORES: GÉNERO, EDAD Y ÁREA DE CONOCIMIENTO}

Los resultados obtenidos en el ANOVA para el caso de la importancia atribuida al diseño y desarrollo de espacios de aprendizaje que favorezcan el respeto y la igualdad de género no mostraron diferencias significativas para ninguno de los factores incluidos. Sin embargo, sí muestran diferencias entre ambos géneros y entre ambas macro-áreas en el caso del nivel de desarrollo adquirido en el diseño y desarrollo de espacios de aprendizaje que favorezcan el respeto y la igualdad de género (Tabla 3).

TABLA 3

RESULTADOS DEL ANOVA PARA LA VARIABLE NIVEL DE DESARROLLO

\begin{tabular}{l|c|c|c|c}
\hline \multicolumn{1}{c|}{ VARIABLES } & Sc & gl & $\boldsymbol{F}$ & $\boldsymbol{p}$ \\
\hline Modelo & 13,67 & 4 & 2,98 & 0.01 \\
Edad & 1,68 & 2 & 0,73 & 0,48 \\
Género & 5,90 & 1 & 5,15 & 0,02 \\
Macro-área & 4,66 & 1 & 4,07 & 0,04 \\
\hline Error & 398,45 & 348 & & \\
\hline Total & 431,12 & 352 & & \\
\hline
\end{tabular}

gl = grados de libertad del denominador; $S c=$ Suma de cuadrados

Fuente: Elaboración propia.

Los resultados del análisis a posteriori (LSD de Fisher) indican diferencias entre los dos géneros (Tabla 4), mostrando que las mujeres asignan un mayor valor al nivel de desarrollo adquirido que los hombres. Por otro lado, este análisis también muestra que los encuestados del macro-área de sociales y humanidades asignan un mayor valor al nivel de desarrollo adquirido a tal competencia que el alumnado de Ciencias y Tecnologías.

TABLA 4

RESULTADOS DEL LSD DE FISHER PARA LA VARIABLE NIVEL DE DESARROLLO

\begin{tabular}{c|c|c|c|c}
\hline MACRO - ÁREA & MEDIAS & $\mathbf{n}$ & E.E. & LETRA \\
\hline Ciencias y Tecnologías & 3,07 & 178 & 0,08 & A \\
\hline Sociales y Humanidades & 3,30 & 175 & 0,09 & B \\
\hline
\end{tabular}

\begin{tabular}{c|c|c|c|c}
\hline SEXO & MEDIAS & $\mathbf{n}$ & E.E. & LETRA \\
\hline Hombres & 3,05 & 163 & 0,09 & $\mathrm{~A}$ \\
\hline Mujeres & 3,31 & 190 & 0,08 & $\mathrm{~B}$ \\
\hline
\end{tabular}

Medias con una letra común no son significativamente diferentes $(p>0,05)$

Error: 1,1450; gl: 348

Fuente: Elaboración propia. 


\section{DISCUSIÓN}

Los resultados parecen indicar de forma clara, en el caso del nivel de desarrollo adquirido entre los estudiantes del Máster MAES, que las variables género y área de conocimiento juegan un papel importante en la determinación de la competencia centrada en el diseño y desarrollo de espacios de aprendizaje que favorezcan el respeto y la igualdad de género.

En la línea de lo que planteaban Forbes y Davis (2010), podemos observar que algunas de las competencias que se atribuyen como menos importantes entre el colectivo, son las menos desarrolladas por los futuros y las futuras docentes (conocer los procesos de interacción del alumnado y la resolución de conflictos, diseñar actividades no formales y el fomento del espíritu crítico, reflexivo y emprendedor). Lo que conllevará una disminución en el tiempo que se dedicará frente a otras competencias que generan un mayor nivel de seguridad. Sin embargo, esto no siempre ocurre; como hemos podido ver, hay competencias que tienen un nivel de importancia atribuido bastante bajo (conocer la normativa del sistema educativo y analizar las características históricas de la profesión) y la percepción sobre el nivel de desarrollo es bastante elevada.

Por otro lado, a diferencia de los resultados de González (2016), el futuro profesorado de Educación Secundaria sí valora como importante la inclusión de espacios y actividades en el aula que fomenten la igualdad de género. Aunque como indican Ukpokodu (2016) y Walton y colaboradores (2018), es necesario seguir reforzando la formación en prácticas relacionadas con la igualdad de género que contribuyan a mejorar el nivel de desarrollo adquirido (GARCÍA et al., 2013).

En otra línea, cuando tenemos en cuenta la formación previa recibida, observamos en los resultados como ésta ejerce una influencia significativa en relación al nivel de desarrollo adquirido. Es decir, como ya anticipaban Goldman y Warren (1973), Watkins y Hattie (1985) y Delgado y Prieto (1993) y más recientemente Harlen (2015), la formación recibida en las diferentes carreras universitarias va a plantear contextos de aprendizaje heterogéneos y como consecuencia, distintos tipos de conductas y enfoques de aprendizaje diferentes en los futuros y las futuras docentes. Resultados muy en consonancia con Cano (2000), cuando afirma que esas diferencias afloran sobre todo cuando entra en juego la formación previa y el género, como ocurre en nuestro estudio.

De acuerdo con los resultados de Díaz de Grenu y Anguita (2017), en nuestro estudio también se evidencia una tendencia femenina para incorporar el diseño de prácticas educativas sobre género en el aula. Por tanto, la sensibilización y formación son imprescindibles en los procesos de inclusión de la perspectiva de género en los contenidos y prácticas educativas (REBOLLO CATALÁN; VEGA CARO; GARCÍA-PEREZ, 2011).

\section{CONCLUSIÓN}

Como formadores y formadoras, conocer las actitudes del profesorado hacia el diseño y desarrollo de espacios que favorezcan el respeto y la igualdad de género 
puede ayudarnos no solo a desarrollar programas formativos específicos para trabajar con nuestro alumnado (futuros y futuras docentes), sino también para conocer cómo perciben este tema y su importancia en la educación futura de sus discentes.

En general, la puesta en marcha del Máster FPES en nuestro país durante los últimos años, junto con la necesidad de transformar la formación inicial del profesorado de secundaria en un proceso que permita disponer de docentes mejor preparados y capacitados para la educación del siglo XXI, ha generado muchas expectativas sobre el nuevo modelo de formación. Con este tipo de trabajos, podemos contribuir a mejorar la FIPS desarrollando investigaciones educativas que ayuden a conocer el pensamiento inicial de los futuros y las futuras docentes, a identificar necesidades formativas, a indagar en las expectativas sobre el proceso de formación inicial, a valorar las competencias docentes que logran desarrollar durante dicho proceso, entre otras.

Como hemos podido concluir en los resultados, variables contextuales como la carrera universitaria estudiada inciden significativamente en las percepciones que los futuros y las futuras docentes tienen sobre la formación recibida y en interacción con el género. Esto implica que no se debe obviar esta variable en espacios de investigación, intervención y enseñanza. Como apuntaban Rebollo Catalán, Vega Caro y García-Perez (2011), es necesario que el profesorado asuma un papel activo, reflexionando y revisando sus actitudes y prácticas docentes. Como anticipábamos en la literatura y en consonancia con Esen (2013), si las políticas educativas promueven la igualdad de género en la educación, la resistencia, la sensibilización y las nuevas prácticas docentes desempeñarían nuevos criterios, roles y actitudes con respecto a la igualdad de género.

Finalmente, consideramos que sería necesario profundizar en los mecanismos que propician estas diferencias y en la evolución de los diferentes estilos por titulación y género. Determinar si las diferencias se deben a factores previos o son determinados por procesos específicos de las diferentes titulaciones. O bien, como se anticipaba en la literatura, si esto puede ser debido al propio sistema de evaluación que se utiliza (LEDER; FORGASZ, 2018). Esto permitiría una mayor comprensión de los estilos de aprendizaje del alumnado y, por ende, de sus procesos de aprendizaje.

\section{REFERENCIAS}

ARRIBAS, J. M.; MANRIQUE, J. C.; TABERNERO, B. Instrumentos de evaluación utilizados en la formación inicial del profesorado y su coherencia para el desarrollo de competencias profesionales en los estudiantes: visión del alumnado, egresados y profesorado. Revista Complutense de Educación, Madrid, v. 27, n. 1, p. 237-255, 2016.

BALZARINI, M.; CASANOVES, J. A.; DI RIENZO, L.; GONZÁLEZ, C.; ROBLEDO, W.; TABLADA, E. InfoStat, versión 1, 1, Manual del Usuario. Grupo InfoStat, FCA, Universidad Nacional de Córdoba. Córdoba, Argentina: Editorial Brujas, 2002.

CANO, F. Diferencias de género en estrategias y estilos de aprendizaje. Psicothema, Asturias, v. 12, n. 3 , p. 360-367, 2000. 
DAY, C.; GU, Q. Profesores: vidas nuevas, verdades antiguas. Madrid, España: Narcea. 2012.

DELGADO, A. R.; PRIETO, G. Limitaciones de la investigación sobre las diferencias sexuales en cognición. Psicothema, Asturias, v. 5, n. 2, p. 419-437, 1993.

DÍAZ DE GRENU, S.; ANGUITA, R. Estereotipos del profesorado en torno al género y a la orientación sexual. Revista Electrónica Interuniversitaria de Formación del Profesorado, Zaragoza, v. 20, n. 1, p. 219-232, 2017.

ESEN, Y. Making room for gender sensitivity in pre-service teacher education. European Researcher, v. 6, n. 10, p. 2544-2554, 2013.

ESPAÑA. Ministerio de Educación y Ciencia. Orden ECI/3858/2007, de 27 de diciembre, por el que se establecen los requisitos para la verificación de los títulos oficiales que habiliten para el ejercicio de las profesiones de Profesor de Educación Secundaria, Obligatoria y Bachillerato, Formación Profesional y Enseñanza de Idiomas. Boletín Oficial del Estado, n. 12, 29 diciembre 2007.

FERRADA, D.; VILLENA, A.; DEL PINO, M. ¿Hay qué reformar a los docentes en políticas educativas? Cadernos de Pesquisa, São Paulo, v. 48, n. 167, p. 254-279, jan./mar. 2018.

FORBES, C. T.; DAVIS, E. A. Curriculum design for inquiry: pre-service elementary teachers' mobilization and adaptation of science curriculum materials. Journal of Research in Science Teaching, v. 47, n. 7, p. 820-839, 2010.

GARCÍA, R.; REBOLLO, M. Á.; VEGA, L.; BARRAGÁN, R.; BUZÓN, O.; PIEDRA, J. El patriarcado no es transparente: competencias del profesorado para reconocer la desigualdad. Cultura y Educación, v. 23, n. 3, p. 385-397, 2011.

GARCÍA, R.; SALA, A.; RODRÍGUEZ, E.; SABUCO, A. Formación inicial del profesorado sobre género y coeducación: impactos metacognitivos de la inclusión curricular transversal sobre sexismo y homofobia. Profesorado: Revista de Currículum y Formación del Profesorado, v. 17, n. 1, p. 269-287, 2013.

GOLDMAN, R.; WARREN, R. Discriminant analysis of study strategies connected with college grades success in different major fields. Journal of Educational Measurement, v. 10, n. 1, p. 39-47, 1973.

GONZÁLEZ, T. Políticas educativas igualitarias en España: la igualdad de género en los estudios de magisterio. Archivo Analítico de Políticas Educativas, v. 26, n. 2, p. 1-20, 2016.

GONZÁLEZ, J.; WAGENAAR, R. Tuning educational structures in Europe II: la contribución de las universidades al Proceso de Bolonia. Bilbao, Spain: Universidad de Deusto, 2006.

GRAHAM, M. H.; EDWARDS, M. S. Statistical significance versus fit: estimating the importance of individual factors in ecological analysis of variance. Oikos, v. 93, n. 3, p. 505-513, June 2001.

HANNON, B. Test anxiety and perfomance-avoidance goals explain gender differences in SAT-V, SAT-M, and overall SAT scores. Personality and Individual Differences, v. 53, n. 7, p. 816-820, 2012.

HARLEN, W. Primary teachers' understanding in science and its impact in the classroom. Research in Science Education, v. 27, n. 3, p. 323-337, 2015.

IZADINIA, M. Preservice teachers' professional identity development and the role of mentor teachers. International Journal of Mentoring and Coaching in Education, v. 5, n. 2, p. 127-143, 2016.

LE HUU NGHIA, T.; NGOC TAI, H. Preservice teachers' identity development during the teaching internship. Australian Journal of Teacher Education, v. 42, n. 8, p. 1-15, Aug. 2017. http://dx.doi.org/10.14221/ ajte.2017v42n8.1

LEDER, G.; FORGASZ, H. J. Measuring who counts: gender and mathematics assessment. ZDM Mathematics Education, v. 50, n. 4, p. 1-11, 2018.

LINDBERG, S. M.; HYDE, J. S.; PETERSEN, J. L.; LINN, M. C. New trends in gender and mathematics performance: a meta-analysis. Psychological Bulletin, v. 136, n. 6, p. 1123-1135, 2010. 
LORENZO VICENTE, J. A.; MUÑOZ GALIANO, I. M.; BEAS MIRANDA, M. Modelos de formación inicial del profesorado de educación secundaria en España desde una perspectiva Europea. Revista Complutense de Educación, v. 26, n. 3, p. 741-757, 2015.

MILLER, C. D.; FINLEY, J.; MCKINLEY, D. L. Learning approaches and motives: male and female differences and implications for learning assistance programs. Journal of College Student Development, v. 3, n. 2, p. 14-32, 1990.

REBOLLO CATALÁN, M. A.; VEGA CARO, L.; GARCÍA-PÉREZ, R. El profesorado en la aplicación de planes de igualdad: conflictos y discursos en el cambio educativo. RIE: Revista de Investigación Educativa, v. 29, n. 2, p. 311-323, 2011.

SÁNCHEZ, A.; LÓPEZ, M. A.; FERNÁNDEZ, M. V. Analysis of the generic competences in the new degrees of the ESEA in the Spanish universities. Revista de Docencia Universitaria, v. 8, n. 1, p. 35-73, 2010.

SERRANO, R.; PONTES, A. Expectativas ante la formación inicial entre el alumnado del Máster de Profesorado de Enseñanza Secundaria. Revista de Investigación Educativa, v. 33, n. 2, p. 489-505, 2015

SEVERIENS, S.; TEN DAM, G. Gender differences in learning styles: a narrative review and quantitative meta-analysis. Higher Education, v. 27, p. 487-501, 1994.

UNESCO. E2030: Education and skills for the 21st century. Santiago, Chile: Orealc/Unesco, 2017.

UKPOKODU, O. Realizing transformative learning and social justice education: unpacking teacher education practice. In: TOMLINSON-CLARKE, S. M.; CLARKE, D. L. (ed.). Social Justice and Transformative Learning: culture and identitty in the United States and South Africa. New York: Routledge, 2016. (Routledge Research in Educational Equality and Diverstity). p. 113-143.

VILLA, A.; POBLETE, M. Evaluación de competencias genéricas: principios, oportunidades y limitaciones. Revista Bordón, v. 63, n. 1, p. 147-170, 2011.

WALTON, J. L.; ROD, S.; FLORY, S.; HILL, J.; SUTHERLAND, S.; FLEMONS, M. Implicit and explicit pedagogical practices related to sociocultural issues and social justice in physical education teacher education programs. Physical Education and Sport Pedagogy, v. 23, n. 5, p. 497-509, 2018.

WATKINS, D.; HATTIE, J. A longitudinal study of the approaches to learning of Australian tertiary students. Human Learning, v. 4, p. 127-141, 1985.

WILSON, K. L.; SMART, R. M.; WATSON, R. J. Gender differences in approaches to learning in first year psychology students. British Journal of Educational Psychology, v. 66, p. 59-71, 1996.

WORLD BANK. Learning to realize education's promise. Washington: World Bank Group, 2018.

ZABALZA, M. A. Evaluación de los planes de formación docente de las universidades. Educar, Barcelona, v. 47, n. 1, p. 181-197, enero/jun. 2011.

NOTA: Rocío Serrano Rodríguez planificó el diseño y la toma de datos, la introducción teórica y la discusión; Elisa Pérez Gracia revisó y completo el marco teórico, los análisis de resultados y las conclusiones; Monserrat Bustamante Chan realizó la revisión del análisis de los datos y la adaptación del texto al estilo y normas de la revista; Antonio Carpio Camargo se encargo del tratamento metodológico de los datos.

\section{COMO CITAR ESTE ARTÍCULO}

SERRANO RODRÍGUEZ, Rocío; PÉREZ GRACIA, Elisa; BUSTAMANTE CHAN, Monserrat; CARPIO CAMARGO, Antonio. Respeto e igualdad de género en la formación inicial docente. Cadernos de Pesquisa, São Paulo, v. 49, n. 174, p. 152-166, out./dez. 2019. https://doi.org/10.1590/198053146162 